\title{
Correction to: Strengthening medical training programmes by focusing on professional transitions: a national bridging programme to prepare medical school graduates for their role as medical interns in Botswana
}

\author{
Michael J. Peluso ${ }^{1,2,3^{*}}$, Rebecca Luckett ${ }^{1,3,4,5}$, Savara Mantzor ${ }^{6,7,8}$, Alemayehu G. Bedada ${ }^{9}$, Paul Saleeb ${ }^{10,11}$, \\ Miriam Haverkamp ${ }^{12}$, Mosepele Mosepele ${ }^{12}$, Cecil Haverkamp ${ }^{13}$, Rosa Maoto ${ }^{14}$, Detlef Prozesky ${ }^{14,15}$, \\ Neo Tapela1,2,3,12,16, Oathokwa Nkomazana ${ }^{14,17}$ and Tomer Barak ${ }^{1,3,14,18,19}$
}

\section{Correction}

Following publication of the original article [1], the first author reported that there was a typographical error in the name of one of his co-authors. The correct spelling is Alemayehu Bedada, not Alemayhu Bedada.

The original article has been updated.

\begin{abstract}
Author details
${ }^{1}$ Botswana-Harvard Partnership, Gaborone, Botswana. ${ }^{2}$ Department of Medicine and Division of Global Health Equity, Brigham and Women's Hospital, Boston, MA, USA. ${ }^{3}$ Harvard Medical School, Boston, USA. ${ }^{4}$ Department of Obstetrics and Gynecology, Scottish Livingstone Hospital, Molepolole, Botswana. ${ }^{5}$ Department of Obstetrics and Gynecology, Beth Israel Deaconess Medical Center, Boston, USA. ${ }^{6}$ Children's Hospital of Philadelphia, Philadelphia, USA. 'Department of Paediatrics, Princess Marina Hospital, Gaborone, Botswana. ${ }^{8}$ Botswana-UPenn Partnership, Gaborone, Botswana. ${ }^{9}$ Department of Surgery, Faculty of Medicine, University of Botswana, Gaborone, Botswana. ${ }^{10}$ University of Maryland, College Park, MD, USA. "Botswana-University of Maryland School of Medicine Health Initiative, Gaborone, Botswana. ${ }^{12}$ Department of Medicine, Princess Marina Hospital, Gaborone, Botswana. ${ }^{13}$ University Research Co, Gaborone, Botswana.

${ }^{14}$ Medical Internship Training Programme, Gaborone, Botswana.

${ }^{15}$ Department of Medical Education, University of Botswana, Gaborone, Botswana. ${ }^{16}$ Botswana Ministry of Health, Gaborone, Botswana. ${ }^{17}$ Faculty of Medicine, University of Botswana, Gaborone, Botswana. ${ }^{18}$ Department of Medicine, Scottish Livingstone Hospital, Molepolole, Botswana. ${ }^{19}$ Department of Medicine, Beth Israel Deaconess Medical Center, Boston, USA.
\end{abstract}

Received: 20 March 2018 Accepted: 20 March 2018 Published online: 27 March 2018

\section{Reference}

1. Peluso MJ, Luckett R, Mantzor S, Bedada AG, Saleeb P, Haverkamp M, Mosepele M, Haverkamp C, Maoto R, Prozesky D, Tapela N, Nkomazana O, Barak T. Strengthening medical training programmes by focusing on professional transitions: a national bridging programme to prepare medical school graduates for their role as medical interns in Botswana. BMC Med Educ. 2017;17:261. https://doi.org/10.1186/s12909-017-1102-1.

* Correspondence: michael.peluso@gmail.com

'Botswana-Harvard Partnership, Gaborone, Botswana

${ }^{2}$ Department of Medicine and Division of Global Health Equity, Brigham and Women's Hospital, Boston, MA, USA 